\title{
Diuretic use, acute kidney injury, and premature infants: the call for evidence-based guidelines
}

\author{
Jeffrey Segar ${ }^{1}$ (D) Jennifer G. Jetton ${ }^{2}$ (D) \\ Received: 22 June 2021 / Accepted: 23 June 2021 / Published online: 13 July 2021 \\ (C) IPNA 2021
}

\section{Introduction}

Acute kidney injury (AKI) is a common morbidity among infants cared for in the neonatal intensive care unit (NICU) and has been recognized to be associated with increased length of stay, risk of chronic kidney injury, and mortality. A series of publications derived from the Assessment of Worldwide Acute Kidney injury Epidemiology in Neonates (AWAKEN) study, a multicenter, retrospective cohort study of ill neonates, has provided a wealth of knowledge regarding the epidemiology of AKI in this population [1-3]. Notably, infants with AKI had a four-times higher independent odds of death and longer hospital length of stay than those without AKI [1]. Moreover, recent studies suggest AKI is a risk factor for the development of chronic kidney disease later in life [4, 5]. However, as evidence accrues regarding the role that AKI plays in the morbidity and mortality of critically ill neonates and infants, we continue to lack robust evidence-based guidelines for prevention and mitigation of this life-threatening event.

To understand the impact of AKI on the health of babies and ultimately to improve outcomes, we need a better appreciation of the risk factors for the development of AKI as well as current management approaches for infants once AKI has occurred. The management of oliguria and fluid overload is a key aspect of care of babies with AKI, and diuretics are often used in this context to promote urine output. Indeed, as shown in an article in this issue of Pediatric Nephrology by Mohamed and colleagues [6], the (off-label) use of diuretics in premature infants with $\mathrm{AKI}$ is common, occurring in $76 \%$

Jennifer G. Jetton

Jennifer-jetton@uiowa.edu

1 Departments of Pediatrics and Physiology, Medical College of Wisconsin, Milwaukee, WI, USA

2 Stead Family Department of Pediatrics, University of Iowa, Iowa City, IA, USA of premature infants with $\mathrm{AKI}$ in their cohort and with considerable variation in prescribing practices across centers. That such broad application occurs in the absence of either evidence-based clinical guidelines for use or robust outcomes data shines a light on an important area for improvement in the care of this vulnerable population.

\section{Understanding the use of diuretics in neonates and infants with AKI}

Mohamed and colleagues have utilized the Pediatric Hospital Information System (PHIS) database to enlighten providers regarding the utilization of diuretics in preterm infants with AKI [6]. Review of records from over 70,000 infants $<37$ weeks gestation and admitted within the first week of life identified 2379 infants with AKI, using the International Classification of Disease, Tenth Revision, code N17 (acute kidney failure, unspecified). The severity or stage of AKI was not available nor were specific creatinine values. Multiple demographic variables were available and included in the analysis, as were diagnoses of oliguria/anuria and highrisk conditions associated with AKI (e.g., congenital heart disease and genitourinary structural abnormalities). As mentioned above, 76\% (1801/2379) of infants with AKI received at least one dose of diuretics while only $16 \%(11,075 / 69,242)$ of those without a diagnosis of AKI were treated with this medication class. Among infants with AKI, treatment with diuretics was associated with younger gestational age and lower birthweight. For infants receiving diuretics (and 99\% of these received furosemide), the median duration was 18 days, with slightly over half of these receiving diuretics for $\geq 5$ consecutive days. In neonates with AKI, treatment with diuretics was significantly associated with increased mortality, need for mechanical ventilation, and length of stay. Interhospital variation in the use of diuretics was also high, with diuretics prescribed to less than half of patients with AKI in some hospitals to almost all patients with AKI in others 
(range 42-96\%). While infants with AKI received diuretics at a far higher rate than those without AKI, the authors could not ascertain the reasons for diuretic administration or the timing of occurrence of AKI with dosing of diuretics. For example, infants with developing or established chronic lung disease may have been treated with diuretics for pulmonary purposes, and not in response to a diagnosis of AKI. The authors appropriately highlight that there are many questions to answer based on the data they have presented in this paper.

\section{Is there a rationale for the widespread use of diuretics?}

The relatively high use of diuretics in the NICU population has previously been established. In a review of a large national dataset (Pediatrix Medical Group data warehouse) from 1996 to 2005 , Clark et al. reported furosemide to be the $7^{\text {th }}$ most commonly prescribed drug in the NICU, with $8-9 \%$ of NICU patients receiving at least one dose [7]. A later analysis of this same database (1997-2011) found that 37\% (39,357/107,542) of infants $<32$ weeks gestation and $<1500 \mathrm{~g}$ birth weight were exposed to at least one diuretic; furosemide was the most commonly used (93\% with $\geq 1$ recorded dose) [8]. Using the same PHIS database as in the current study, Bamat et al. identified that for infants $<32$ weeks gestation with grade 2 or 3 bronchopulmonary dysplasia, almost $95 \%$ of infants were exposed to loop diuretics at least once during hospital admission, with the duration of use ranging from 7.3 to $49.4 \%$ of all hospital days [9]. Neither these nor other studies have been able to establish that use of diuretics improves short- or longterm outcomes.

While rationale, or lack thereof, for the use of diuretics in infants with chronic lung disease has been discussed elsewhere, their use in infants with AKI warrants additional consideration $[10,11]$. Several studies have shown that neonatal fluid overload is associated with increased morbidity and mortality [12] including in infants supported with extracorporeal membrane oxygenation (ECMO) and following cardiac surgery $[13,14]$. In addition, a positive fluid balance in the first week of life has been associated with increased risk of requiring mechanical ventilation (MV) in both preterm ( $<36$ weeks) and near-term/term ( $\geq 36$ weeks) neonates $[2,15]$ and increased risk of patent ductus arteriosus [16]. Thus, efforts to promote diuresis and maintain fluid balance in infants with AKI appear justified. Certainly, for the smallest patients, diuretics may represent the only option available for fluid removal given the lack of appropriately sized dialysis technology.

Practitioners may also prescribe furosemide in the setting of AKI in an attempt to ameliorate the severity of injury by increasing renal blood flow through the stimulation of prostaglandins and decreasing renal oxygen consumption $[17,18]$.
Renal oxygen consumption is proportional to renal tubular sodium reabsorption. It has been estimated that approximately $80 \%$ of renal oxygen consumption is related to activity of the $\mathrm{Na}-\mathrm{H}$ antiporter [19]. While data from pediatric populations are missing, studies from animals and human adults may prove informative. In a sheep model of septic AKI, furosemide significantly increased fractional excretion of sodium (FENa) and renal medullary PO2 without an effect on medullary perfusion, renal blood flow, or renal oxygen delivery, implying decreased oxygen consumption [20]. Despite restoration of medullary $\mathrm{PO} 2$, no sustained improvement in kidney function, including creatinine clearance, FENa, or urine volume, was seen. In adults following cardiopulmonary bypass, furosemide significantly increased FENa and urine output, and decreased tubular sodium reabsorption, associated with a $23 \%$ decrease in renal oxygen consumption [18]. However, glomerular filtration rate decreased by $12 \%$, possibly by feedback mechanisms resulting in constriction of afferent arterioles. Furosemide has additionally been shown in cardiac surgery patients with normal kidney function to decrease creatinine clearance [21]. Taken together, while experimental data suggest that renal workload may be reduced by diuretics, there is no evidence that diuretic administration can prevent or decrease the severity of AKI. Clinical studies in adult cohorts have shown this finding to be true as well [22-24].

\section{Diuretics and outcomes-what is the relationship?}

An additional finding to note in the report of Mohamed et al. was the increased mortality in those infants receiving diuretics. At all gestational ages, infants who survived to and were treated with either a short or long course of diuretics at or before 28 days had lower probability of survival compared to those who did not receive diuretics before 28 days. Reasons for this relationship were not able to be explored given the nature of the investigation. The authors acknowledge, as have others who have identified a similar relationship, that diuretic use may be a marker of severity of illness, being administered to those infants with severe oliguria/anuria or fluid overload. However, in a recent retrospective analysis of 456 patients admitted to a pediatric intensive care unit, of whom $43.4 \%$ received furosemide in the first week of admission, Dai et al. reported mortality was twice that in the furosemide-treated group, even after adjusting for severity of illness [25]. In contrast, Zhao et al. used a large, adult intensive care database to match 4427 pairs of patients with AKI who received furosemide and those without diuretics treatment [26]. Furosemide was associated with reduced in-hospital mortality [hazard ratio (HR) 0.67 ; 95\% CI 0.61-0.74; P < 0.001] and 90-day mortality [HR $0.69 ; 95 \%$ CI $0.64-0.75 ; \mathrm{P}<0.001$ ] in overall AKI patients, though this relationship was not seen in all 
subgroups. The authors note that the reduction in mortality was not seen in patients with AKI stage 2-3 according to serum creatinine (rather than urine output) criteria, in patients with mild AKI ( $0-1$ by urine output criteria), and in those with acute-on-chronic kidney injury. The disparate findings within and across studies support the need for additional rigorous investigation specifically in neonatal and infant cohorts with attention to the AKI stages and definitions, biomarkers used to define AKI, fluid balance, and associated comorbidities identified. Benefit (or harm) may be restricted to subsets of patients with AKI (severity, oliguric vs. non-oliguric, fluid overload, etc.) that will need to be more clearly defined to optimize diuretic use.

\section{Beyond diuretics}

While kidney support therapy (KST) is used more broadly in older children and adults with severe AKI, oliguria, and fluid overload, the use of KST is much less common in the NICU setting. Indeed, in the AWAKEN study, KST was used in only $4 \%(25 / 605)$ of patients with AKI [1]. Peritoneal dialysis (PD) remains a safe and effective kidney support option for babies with both $\mathrm{AKI}$ and $\mathrm{CKD}$, and novel techniques like continuous flow PD may offer more options to provide this therapy safely to some of the smallest babies [27]. In addition, low extracorporeal volume dialysis machines designed specifically for babies as small as $2.5 \mathrm{~kg}$ are gradually becoming more available [28]. These new systems may change the risk/benefit profile of heavy diuretic use in those with the most severe AKI. Of course, the provision of dialysis also carries risk (e.g., risks to long-term vascular access options in patients who have vascular catheters placed at such an early age) and higher costs, and will not be available to all babies outside of highly resourced settings. Again, it will be important to study the impact and use of these therapies on outcomes, especially morbidity, mortality, hospital length of stay, and long-term kidney function.

\section{Rational use of diuretics: consideration for quality improvement initiatives}

Clearly, the use of diuretics is a mainstay of care of the most critically ill patients in all intensive care units. While the association between diuretic use and mortality as demonstrated in this and other studies may be considered simply a "marker of illness severity," the fact that this medication class is so intricately related with critical illness, mortality, and highrisk conditions suggests that closer scrutiny and more dedicated study is warranted, especially in light of conflicting clinical evidence as shown above. Neonatal intensive care unit therapy has seen tremendous advances and improvement in care through commitment to evidence-based care protocols that are subject to rigorous evaluation and study. The use of diuretics, as suggested by the data in this study, has not seen the same level of attention except in specific conditions such as bronchopulmonary dysplasia. The question of whether or not diuretics cause harm (or at least do not offer meaningful benefit) in the context of neonatal and infant AKI should be addressed more intentionally.

Because of the lack of proven treatments to reverse the course of AKI once it has occurred, more and more attention is being paid to preventive strategies. Moreover, these strategies can be framed in the context of quality improvement efforts whereby they can be tracked, critically appraised, and revised. Harer et al. recently published a well-framed neonatal response to the Acute Disease/Dialysis Quality Initiative (AQDI) outlining NICU-specific AKI quality improvement guidelines and approaches [29]. These include a neonatal "AKI bundle" that lists monitoring fluid intakes, outputs, daily balance, and percent fluid accumulation as key components for the evaluation of potentially modifiable risks and complications of AKI [29]. All of these parameters should be taken into account in assessments of effective diuretic use. In addition, the Nephrotoxic Injury Negated by Just-in-time Action (NINJA) project [30] and its NICU corollary Baby NINJA [31] showed that it is possible to reduce nephrotoxic medication-related AKI frequency and severity by careful assessment of antimicrobial need, combination dosing, and creatinine surveillance, as well as the promotion of alternative, non-nephrotoxic medications using a multidisciplinary team approach and strategic use of the electronic medical record. The use of diuretics should be evaluated similarly through a quality lens - one that includes the context of fluid overload, concomitant use of other potentially nephrotoxic drugs, serum creatinine surveillance, and other AKI mitigation strategies. In this way, specific indications for, duration of use, and timing of diuretic prescription could be developed and optimized specifically for neonatal care. Many additional questions about diuretic use in neonates, including but not limited to the identification of the most effective diuretic(s), the tracking and monitoring of diuretic complications (e.g., electrolyte abnormalities, impact on hearing, bone health, and worsening of AKI), strategies to mitigate fluid overload, and the appropriate time to consider KST instead of diuretics, need to be addressed.

Until further data are available, prudent use of diuretics for neonatal AKI is likely appropriate to address fluid overload, especially in the smallest patients for whom no KST options exist. In such cases, higher doses than typically administered may be necessary. Additionally, furosemide may have a role in the treatment of hyperkalemia. In the absence of such conditions, and with the understanding that diuretics themselves have little benefit to kidney function itself (i.e., diuretics cannot "kick-start" kidney function), harm from diuretic 
administration may outweigh potential benefit, and constant attention to response and emerging side effects should be part of the care. At the least, the outcomes of choices regarding diuretic use, both positive and negative, should be examined carefully such that rational decisions can be made and robust evidence-based clinical guidelines can be developed.

\section{Conclusion}

Diuretics are widely used in critically ill and high-risk patients. Mohamed et al. have documented high use in premature babies with AKI compared with those who did not have AKI. Mortality rates and other morbidities were higher in babies who received diuretics and who had AKI, though this is not to imply causation. Additional scrutiny is needed to develop rational and evidence-based practices to optimize outcomes and minimize side effects of this medication class that is widely used in the care of critically ill neonates and infants.

Author contribution Drs. Segar and Jetton contributed equally to the conception and writing of this editorial commentary. Dr. Segar wrote the first draft. Dr. Jetton added additional content, then both authors offered revisions. Both authors read and approved the final manuscript.

Funding The authors did not receive support from any organization for the submitted work.

Data availability No unique data or material was used in the preparation of this manuscript.

\section{Declarations}

Ethics approval This is an editorial commentary. No ethics approval was required.

Consent to participate This work did not involve the participation of human subjects. Therefore, no consent for publication is needed.

Consent for publication This work did not involve the participation of human subjects. Therefore, no consent for publication is needed.

Conflict of interest The authors declare no competing interests.

\section{References}

1. Jetton JG, Boohaker LJ, Sethi SK, Wazir S, Rohatgi S, Soranno DE, Chishti AS, Woroniecki R, Mammen C, Swanson JR, Sridhar S, Wong CS, Kupferman JC, Griffin RL, Askenazi DJ; Neonatal Kidney Collaborative (NKC) (2017) Incidence and outcomes of neonatal acute kidney injury (AWAKEN): a multicentre, multinational, observational cohort study. Lancet Child Adolesc Health 1: 184-194

2. Selewski DT, Akcan-Arikan A, Bonachea EM, Gist KM, Goldstein SL, Hanna M, Joseph C, Mahan JD, Nada A, Nathan AT, Reidy K,
Staples A, Wintermark P, Boohaker LJ, Griffin R, Askenazi DJ, Guillet R, Neonatal Kidney Collaborative (2019) The impact of fluid balance on outcomes in critically ill near-term/term neonates: a report from the AWAKEN study group. Pediatr Res 85:79-85

3. Charlton JR, Boohaker L, Askenazi D, Brophy PD, Fuloria M, Gien J, Griffin R, Hingorani S, Ingraham S, Mian A, Ohls RK, Rastogi S, Rhee CJ, Revenis M, Sarkar S, Starr M, Kent AL; Neonatal Kidney Collaborative (NKC) (2019) Late onset neonatal acute kidney injury: results from the AWAKEN Study. Pediatr Res 85:339-348

4. Chawla LS, Eggers PW, Star RA, Kimmel PL (2014) Acute kidney injury and chronic kidney disease as interconnected syndromes. N Engl J Med 371:58-66

5. Harer MW, Pope CF, Conaway MR, Charlton JR (2017) Follow-up of Acute kidney injury in Neonates during Childhood Years (FANCY): a prospective cohort study. Pediatr Nephrol 32:10671076

6. Mohamed TH, Klamer B, Mahan JD, Spencer JD, Slaughter JL (2021) Diuretic therapy and acute kidney injury in preterm neonates and infants. Pediatr Nephrol. https://doi.org/10.1007/s00467021095132-6

7. Clark RH, Bloom BT, Spitzer AR, Gerstmann DR (2006) Reported medication use in the neonatal intensive care unit: data from a large national data set. Pediatrics 117:1979-1987

8. Laughon MM, Chantala K, Aliaga S, Herring AH, Hornik CP, Hughes R, Clark RH, Smith PB (2015) Diuretic exposure in premature infants from 1997 to 2011. Am J Perinatol 32:49-56

9. Bamat NA, Nelin TD, Eichenwald EC, Kirpalani H, Laughon MM, Jackson WM, Jensen EA, Gibbs KA, Lorch SA (2021) Loop diuretics in severe bronchopulmonary dysplasia: cumulative use and associations with mortality and age at discharge. J Pediatr 231(4349):e3

10. Segar JL (2020) Rethinking furosemide use for infants with bronchopulmonary dysplasia. Pediatr Pulmonol 55:1100-1103

11. Zayegh AM, Davis PG (2021) BPD treatments: the never-ending smorgasbord. Semin Fetal Neonatal Med 26:101223

12. Askenazi DJ, Koralkar R, Hundley HE, Montesanti A, Patil N, Ambalavanan N (2013) Fluid overload and mortality are associated with acute kidney injury in sick near-term/term neonate. Pediatr Nephrol 28:661-666

13. Gorga SM, Sahay RD, Askenazi DJ, Bridges BC, Cooper DS, Paden ML, Zappitelli M, Gist KM, Gien J, Basu RK, Jetton JG, Murphy HJ, King E, Fleming GM, Selewski DT (2020) Fluid overload and fluid removal in pediatric patients on extracorporeal membrane oxygenation requiring continuous renal replacement therapy: a multicenter retrospective cohort study. Pediatr Nephrol 35:871882

14. Wilder NS, Yu S, Donohue JE, Goldberg CS, Blatt NB (2016) Fluid overload is associated with late poor outcomes in neonates following cardiac surgery. Pediatr Crit Care Med 17:420-427

15. Selewski DT, Gist KM, Nathan AT, Goldstein SL, Boohaker LJ, Akcan-Arikan A, Bonachea EM, Hanna M, Joseph C, Mahan JD, Mammen C, Nada A, Reidy K, Staples A, Wintermark P, Griffin R, Askenazi DJ, Guillet R, Neonatal Kidney Collaborative (2020) The impact of fluid balance on outcomes in premature neonates: a report from the AWAKEN study group. Pediatr Res 87:550-557

16. Bell EF, Warburton D, Stonestreet BS, Oh W (1980) Effect of fluid administration on the development of symptomatic patent ductus arteriosus and congestive heart failure in premature infants. N Engl J Med 302:598-604

17. Williamson HE, Bourland WA, Marchand GR, Farley DB, Van Orden DE (1975) Furosemide induced release of prostaglandin E to increase renal blood flow. Proc Soc Exp Biol Med 150:104-106

18. Swärd K, Valsson F, Sellgren J, Ricksten SE (2005) Differential effects of human atrial natriuretic peptide and furosemide on 
glomerular filtration rate and renal oxygen consumption in humans. Intensive Care Med 31:79-85

19. Ricksten SE, Bragadottir G, Redfors B (2013) Renal oxygenation in clinical acute kidney injury. Crit Care 17:221

20. Iguchi N, Lankadeva YR, Mori TA, Osawa EA, Cutuli SL, Evans RG, Bellomo R, May CN (2019) Furosemide reverses medullary tissue hypoxia in ovine septic acute kidney injury. Am J Phys Regul Integr Comp Phys 317:R232-R239

21. Lassnigg A, Donner E, Grubhofer G, Presterl E, Druml W, Hiesmayr M (2000) Lack of renoprotective effects of dopamine and furosemide during cardiac surgery. J Am Soc Nephrol 11:97104

22. Karajala V, Mansour W, Kellum JA (2009) Diuretics in acute kidney injury. Minerva Anestesiol 75:251-257

23. Bagshaw SM, Bellomo R, Kellum JA (2008) Oliguria, volume overload, and loop diuretics. Crit Care Med 36(4 Suppl):S172S178

24. Bagshaw SM, Gibney RTN, Kruger P, Hassan I, McAlister FA, Bellomo R (2017) The effect of low-dose furosemide in critically ill patients with early acute kidney injury: a pilot randomized blinded controlled trial (the SPARK study). J Crit Care 42:138-146

25. Dai X, Chen J, Li W, Bai Z, Li X, Wang J, Li Y (2020) Association between furosemide exposure and clinical outcomes in a retrospective cohort of critically ill children. Front Pediatr 8:589124

26. Zhao GJ, Xu C, Ying JC, Lü WB, Hong GL, Li MF, Wu B, Yao YM, Lu ZQ (2020) Association between furosemide administration and outcomes in critically ill patients with acute kidney injury. Crit Care 24:75

27. Nourse P, Cullis B, Finkelstein F, Numanoglu A, Warady B, Antwi $\mathrm{S}, \mathrm{McCulloch} \mathrm{M}$ (2021) ISPD guidelines for peritoneal dialysis in acute kidney injury: 2020 update (paediatrics). Perit Dial Int 41: $139-157$

28. Garzotto F, Vidal E, Ricci Z, Paglialonga F, Giordano M, Laforgia N, Peruzzi L, Bellettato M, Murer L, Ronco C (2020) Continuous kidney replacement therapy in critically ill neonates and infants: a retrospective analysis of clinical results with a dedicated device. Pediatr Nephrol 35:1699-1705

29. Harer MW, Selewski DT, Kashani K, Basu RK, Gist KM, Jetton JG, Sutherland SM, Zappitelli M, Goldstein SL, Mottes TA, Askenazi DJ (2020) Improving the quality of neonatal acute kidney injury care: neonatal-specific response to the 22nd Acute Disease Quality Initiative (ADQI) conference. J Perinatol 41:185-195

30. Goldstein SL, Dahale D, Kirkendall ES, Mottes T, Kaplan H, Muething S, Askenazi DJ, Henderson T, Dill L, Somers MJG, Kerr J, Gilarde J, Zaritsky J, Bica V, Brophy PD, Misurac J, Hackbarth R, Steinke J, Mooney J, Ogrin S, Chadha V, Warady B, Ogden R, Hoebing W, Symons J, Yonekawa K, Menon S, Abrams L, Sutherland S, Weng P, Zhang F, Walsh K (2020) A prospective multi-center quality improvement initiative (NINJA) indicates a reduction in nephrotoxic acute kidney injury in hospitalized children. Kidney Int 97:580-588

31. Stoops C, Stone S, Evans E, Dill L, Henderson T, Griffin R, Goldstein SL, Coghill C, Askenazi DJ (2019) Baby NINJA (Nephrotoxic Injury Negated by Just-in-Time Action): reduction of nephrotoxic medication-associated acute kidney injury in the neonatal intensive care unit. J Pediatr 215:223-228.e6

Publisher's note Springer Nature remains neutral with regard to jurisdictional claims in published maps and institutional affiliations. 